\title{
'Errors-and-Erasures' Decoding in RS coded DS-CDMA Using Noncoherent $M$-ary Orthogonal Modulation
}

\author{
Lie-Liang Yang, Lajos Hanzo \\ Dept. of ECS, Univ. of Southampton, SO17 1BJ, UK. \\ Tel: +442380593 125, Fax: +442380594508 \\ Email: lh@ecs.soton.ac.uk, http: //www-mobile.ecs.soton.ac.uk
}

\begin{abstract}
In this contribution we investigate the performance of Reed-Solomon (RS) coded DS-CDMA using noncoherent $M$-ary orthogonal modulation over multipath Rayleigh fading channels. 'Errors-and-erasures' RS decoding is considered, where erasures are declared based on various erasure insertion schemes, such as the maximum Output Threshold Test (OTT), Ratio Threshold Test (RTT) and the joint Maximum Output and Ratio Threshold Test (MOR-TT). Furthermore, the performance of 'errors-anderasures' decoding employing the joint MOR-TT is compared to that of 'error-correction-only' decoding and to that of 'errors-and-erasures' decoding using the OTT as well as the RTT. The numerical results show that, when using 'errors-and-erasures' decoding, RS codes of a given code rate can achieve a significantly higher coding gain than without erasure information, and that the MOR-TT technique outperforms both the RTT and the OTT techniques.
\end{abstract}

\section{Introduction}

In Direct Sequence Code-Division Multiple-Access (DSCDMA) cellular systems Forward Error Correction (FEC) coding is often used for mitigating the effects of fading and interference. For the well-known Reed-Solomon (RS) codes, 'errors-and-erasures' decoding is preferable to 'error-correction-only' decoding, since 'errors-anderasures' decoding can typically achieve a significantly lower codeword decoding error probability, than 'errorcorrection-only' decoding, if a reliable erasure insertion scheme is invoked. Hence, in the context of RS decoding it is beneficial to determine the reliability of the received symbols and to erase the low-reliability symbols prior to the decoding process. In the context of 'errorsand-erasures' decoding, the erasure insertion schemes are expected to exhibit low-complexity and low-delay, while achieving as low a codeword decoding error probability as possible.

On the up-link of a DS-CDMA cellular system, due to the high complexity of coherent modulation/demodulation, which would require a pilot signal for each user, noncoherent $M$-ary orthogonal modulation

This work has been carried out in the framework of the IST project IST-1999-12070 TRUST, which is partly funded by the European Union. The authors would like to acknowledge the contributions of their colleagues from Siemens AG, France Télécom - CNET, Centre Suisse d'Electronique et de Microtechnique S.A., King's College London, Motorola Ltd, Panasonic European Laboratories $\mathrm{GmbH}$, Robert Bosch $\mathrm{GmbH}$, Telefonica Investigation Y Desarrollo S.A. Unipersonal, Toshiba Research Europe Ltd., TTI Norte S.L., University of Bristol, University of Southampton.

EUROCON '2001, 5-7 July 2001, Bratislava using $M=64$, i.e. 6 -bit symbols has been proposed for example for the reverse link of IS-95 [1]. Analysis of DS-CDMA systems using $M$-ary orthogonal signaling - both with and without RS FEC - has been provided for example in [2]-[7] over multipath fading channels. In the context of 'errors-and-erasures' decoding of RS coded DS-CDMA using $M$-ary orthogonal modulation, the erasure insertion schemes based on the Output Threshold Test (OTT) [3], [6], [8], Ratio Threshold Test (RTT) [7] as well as joint Maximum Output and Ratio Threshold Test (MOR-TT) [9] constitute attractive, low-complexity and low-delay erasure insertion schemes. In this treatise the above mentioned erasure insertion schemes as well as the erasure insertion scheme based on the Bayesian Test (BT) [10] are first reviewed. Then, the performance of a RS coded DS-CDMA system is investigated, when $M$-ary orthogonal signaling is employed in conjunction with a OTT-, RTT- or MOR-TT-based erasure insertion scheme over dispersive Rayleigh-fading channels. Furthermore, the performance of the associated $M$-ary DS-CDMA system in conjunction with RS codes assisted by 'errors-anderasures' decoding employing the OTT, RTT and MORTT are estimated and compared. We also compare the codeword decoding error performance of the MOR-TT based erasure insertion scheme to that of using 'errorcorrection-only' decoding without side information. Note that, since the complexity of the BT-based erasure insertion scheme is significantly higher than that of the OTT, RTT or MOR-TT, the performance investigation for the RS coded $M$-ary DS-CDMA system using BTbased 'errors-and-erasures' decoding is not considered in this contribution.

\section{System Overview}

\section{A. Transmitted Signal and Channel Model}

The transmitter schematic of the coded DS-CDMA system using $M$-ary orthogonal modulation is shown in Fig.1. In the transmitter of Fig. 1 the information bits are first grouped into $b$-bit symbols, where $b=\log _{2} M$. Then, $\mathcal{K}$ information symbols are encoded into an $\mathcal{N}$-symbol RS codeword. Following RS encoding the codewords are interleaved, in order to randomize the bursty symbol errors. Finally, each RS coded symbol is $M$-ary modulated, DS spread and carrier-modulated using the approach of [2], in order to form the transmitted signal. Let us assume that there are $K$ active users transmitting their signals simultaneously. Then the signal transmitted by user $i$, 


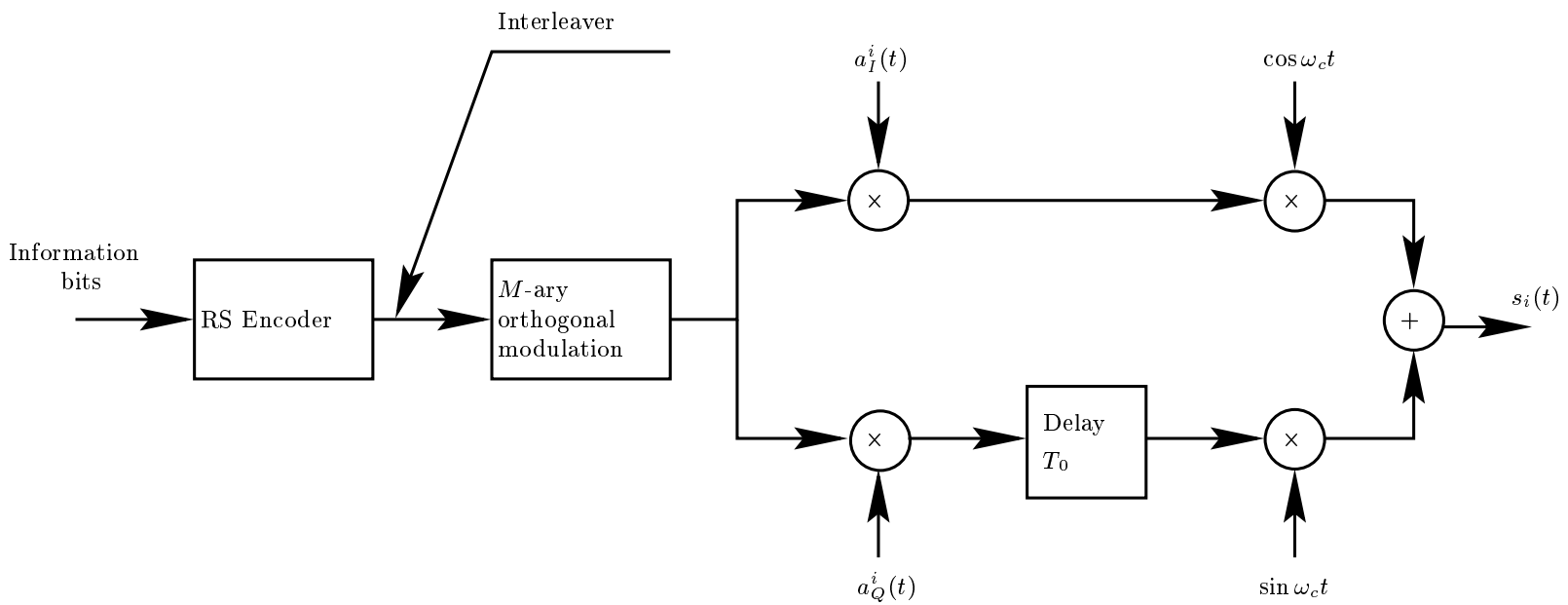

Fig. 1. Transmitter block diagram of the coded DS-CDMA system using $M$-ary orthogonal modulation, RS encoding and symbol interleaving.

$i=1,2, \ldots, K$, can be expressed as [2]

$$
\begin{aligned}
s_{i}(t) & =\sqrt{P R_{c}} W^{j}(t) a_{I}^{i}(t) \cos \omega_{c} t \\
& +\sqrt{P R_{c}} W^{j}\left(t-T_{0}\right) a_{Q}^{i}\left(t-T_{0}\right) \sin \omega_{c} t, 0 \leq t \leq T_{s}
\end{aligned}
$$

where $P$ is the transmitted symbol power without FEC, $R_{c}$ is the coding rate, $T_{s}$ is the symbol duration, $\omega_{c}$ is the carrier's angular frequency and $T_{0}$ is an offset time. Furthermore, $W^{j}(t)$ is the $j$ th Walsh-Hadamard orthogonal function, which represents the $j$ th orthogonal signal of the $i$ th user's symbols, while $a_{I}^{i}(t)$ and $a_{Q}^{i}(t)$ represent the spreading waveforms of the In-phase (I) and Quadrature (Q) phase channels, respectively. These quadrature components are expressed as $a_{\vartheta}^{i}(t)=\sum_{h=-\infty}^{\infty} a_{h}^{\vartheta, i} p\left(t-h T_{c}\right)$ where $\vartheta \in\{I, Q\}, a_{h}^{\vartheta, i}$ represents independent identically distributed (i.i.d) random variables assuming values of +1 and -1 with equal probability of $1 / 2$. Furthermore, $T_{c}$ represents the chip duration, and $p(t)$ is assumed to be the rectangular chip waveform, which is defined over the interval $\left(0, T_{c}\right]$. Moreover, we assume that $N_{s}=T_{s} / T_{c}=b T_{b} / T_{c}=b N$, where $T_{b}$ is the bitduration and $N=T_{b} / T_{c}$.

The dispersive fading channels are assumed to be discrete and time-invariant, having a channel impulse response of $h_{i}(t)=\sum_{l=1}^{L} \alpha_{i l} \delta\left(t-\tau_{i l}\right) \exp \left(-j \phi_{i l}\right)$ experienced by user $i$, where $\alpha_{i l}, \tau_{i l}$ and $\phi_{i l}$ represent the attenuation factor, delay and phase-shift for the $l$ th multipath component of the channel, respectively, while $L$ is the total number of diversity paths and $\delta(t)$ is the Deltafunction. We assume that the $i$ th user's multipath attenuations $\left\{\alpha_{i l}, l=1,2, \ldots, L\right\}$ are independent Rayleighdistributed random variables having a PDF given by

$$
f_{\alpha_{i l}}(R)=\frac{2 R}{\Omega} \exp \left(-\frac{R^{2}}{\Omega}\right)
$$

where $\Omega=E\left[\left(\alpha_{i l}\right)^{2}\right]$. The phases $\left\{\phi_{i l}, l=1,2, \ldots, L\right\}$ of the different paths are assumed to be uniformly distributed random variables in $[0,2 \pi)$, while the $i$ th user's path delays of $\left\{\tau_{i l}, l=1,2, \ldots, L\right\}$ are modeled as random variables that are mutually independent of each other and uniformly distributed in $\left[0, T_{s}\right)$. We also assume that ideal power control is employed, and hence the received 1) signal powers are the same for all $K$ users. Then the re, ceived signal at the base station generated by the $K$ users can be expressed as

$$
r(t)=\sum_{i=1}^{K} y_{i}(t)+n(t)
$$

where

$$
\begin{aligned}
y_{i}(t)= & \sum_{l=1}^{L} \sqrt{P} \alpha_{i l}\left[W^{j}\left(t-\tau_{i l}\right) a_{I}^{i}\left(t-\tau_{i l}\right) \cos \left(\omega_{c}\left(t-\tau_{i l}\right)-\phi_{i l}\right)\right. \\
& \left.+W^{j}\left(t-T_{0}-\tau_{i l}\right) a_{Q}^{i}\left(t-T_{0}-\tau_{i l}\right) \sin \left(\omega_{c}\left(t-\tau_{i l}\right)-\phi_{i l}\right)\right] .
\end{aligned}
$$

Furthermore, $n(t)$ represents the Additive White Gaussian Noise (AWGN), which is modeled as a random variable with zero mean and double-sided power spectral density of $N_{0} / 2$. After the receiver's bandpass filter, $n(t)$ becomes a narrow-band noise process, which can be expressed as $n(t)=n_{c}(t) \cos \omega_{c} t+n_{s}(t) \sin \omega_{c} t$ [2], where $n_{c}(t)$ and $n_{s}(t)$ represent lowpass-filtered Gaussian processes.

\section{B. Receiver Model}

The receiver schematic considered in this paper for the RS coded DS-CDMA system is shown in Fig.2, which includes noncoherent $M$-ary demodulation, equal gain combining (EGC), maximum likelihood detection (MLD), erasure insertion based on the OTT, RTT or MOR-TT, deinterleaving and RS 'errors-and-erasures' decoding. Furthermore, the square-law-based noncoherent $M$-ary demodulation block is the same as that used in [2] and hence the interested readers are referred to [2] for further details. Multipath diversity combining invoking the EGC principle was implemented in the 'EGC' block. The MLD block selects the largest from its input variables and computes the value of $\lambda$ - the ratio of the 'second' maximum to the maximum of the MLD's inputs. Following the MLD stage, the next block may output an $M$-ary RS code symbol or 


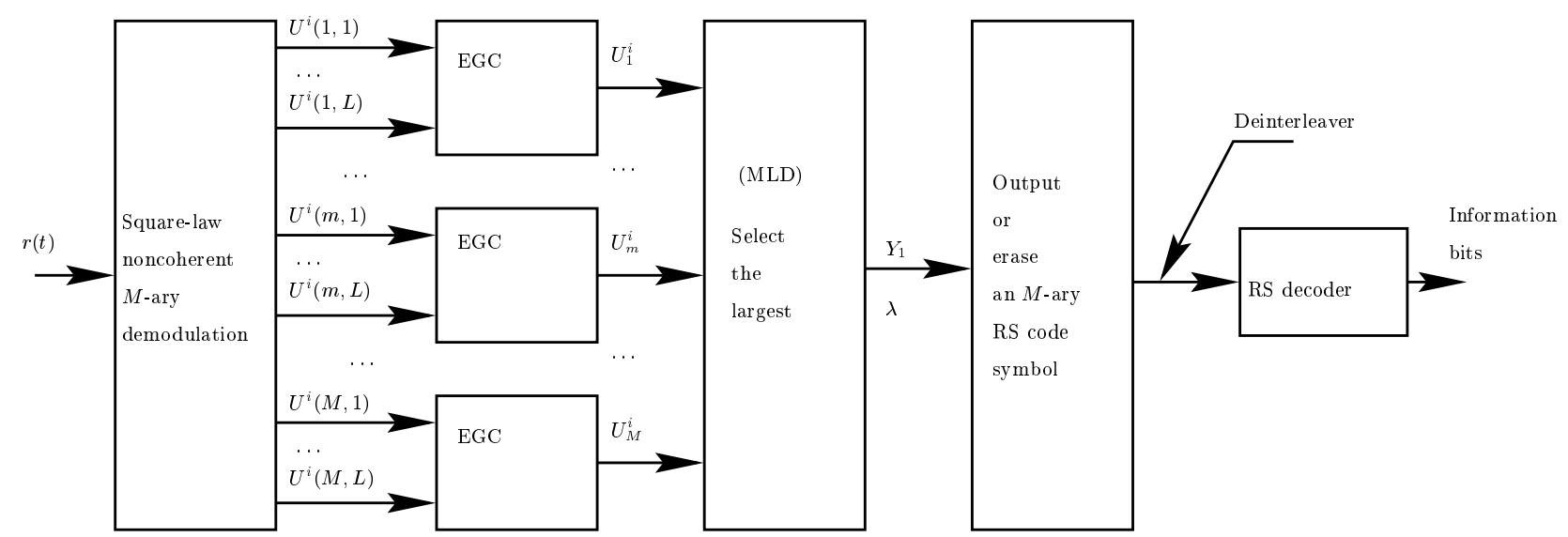

Fig. 2. Receiver model of the RS coded DS-CDMA system investigated using noncoherent $M$-ary demodulation, multipath diversity combining, Maximum-Likelihood Detection (MLD), MOR-TT based erasure insertion, deinterleaving and RS 'errors-and-erasures' decoding.

insert an erasure. Finally, after symbol-based deinterleaving, the RS decoder invokes 'errors-and-erasures' decoding and then outputs the received information bits.

\section{Overview of Erasure Insertion Schemes}

In this section four erasure insertion schemes associated with the noncoherent $M$-ary orthogonal demodulation are reviewed. Let $\left\{U_{1}^{i}, U_{2}^{i}, \ldots, U_{M}^{i}\right\}$ represent the decision variables input to the MLD block of Fig.2. We express the maximum and the 'second' maximum of $\left\{U_{1}^{i}, U_{2}^{i}, \ldots, U_{M}^{i}\right\}$ as

$$
\begin{aligned}
& Y_{1}=\max _{1}\left\{U_{1}^{i}, U_{2}^{i}, \ldots, U_{M}^{i}\right\}, \\
& Y_{2}=\max _{2}\left\{U_{1}^{i}, U_{2}^{i}, \ldots, U_{M}^{i}\right\} .
\end{aligned}
$$

Furthermore, let $H_{1}$ and $H_{0}$ represent the hypotheses of correct decision and erroneous decision concerning an $M$ ary symbol in the MLD block of Fig.2. Hence, we have $P\left(H_{0}\right)=1-P\left(H_{1}\right)$.

\section{A. OTT}

In the context of the OTT, the decision variable subjected to an erasure insertion is $Y_{1}$, i.e., the actual demodulator output is observed. Let $f_{Y_{1}}\left(y \mid H_{1}\right)$ and $f_{Y_{1}}\left(y \mid H_{0}\right)$ be the conditional probability density functions (PDF), given that the associated demodulated symbol is correct $\left(H_{1}\right)$ and incorrect $\left(H_{0}\right)$, respectively. As an example, the distributions of $f_{Y_{1}}\left(y \mid H_{1}\right)$ and $f_{Y_{1}}\left(y \mid H_{0}\right)$ over multipath Rayleigh fading channels are shown in Fig.3, where the diversity order is $L=3$, the average signal-to-noise ratio (SNR) per bit is $\gamma_{b}=5 d B$ and $M=16,64,256$. From the figure we observe that for a given value of $M, f_{Y_{1}}\left(y \mid H_{1}\right)$ is mainly distributed over a normalized demodulator output range associated with relatively high values of $Y_{1}$, while $f_{Y_{1}}\left(y \mid H_{0}\right)$ is spread over a range having relatively low values of $Y_{1}$. Consequently, we can argue that the demodulated symbols having relatively low values of $Y_{1}$ are less reliable than those having relatively high values of $Y_{1}$. Let $Y_{T}$ be a threshold associated with making an erasure decision based on the OTT. Then, if $Y_{1} \leq Y_{T}$, the

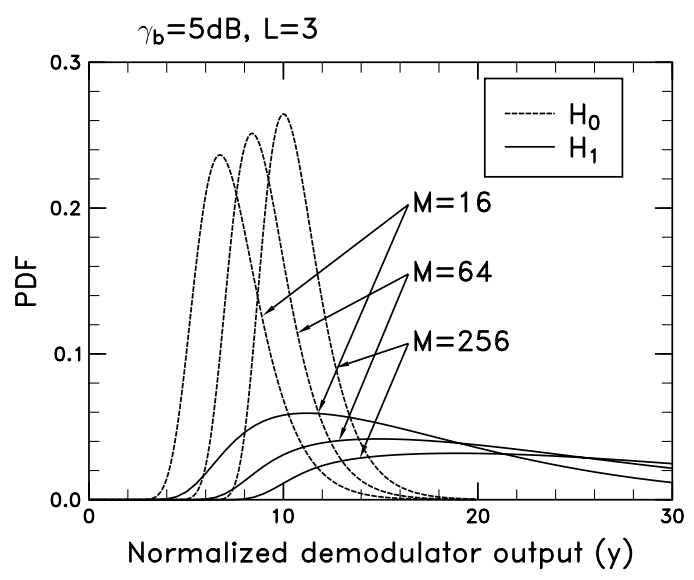

Fig. 3. The PDFs of $Y_{1}=\max \{\cdot\}$ under the hypotheses of $H_{1}$ and $H_{0}$ using $M=16,64,256, L=3, \gamma_{b}=5 \mathrm{~dB}$ over multipath Rayleigh-fading channels.

associated demodulated symbol should be erased. Otherwise, if $Y_{1}>Y_{T}$, the demodulator outputs a RS code symbol.

\section{B. RTT}

The ratio involved in Viterbi's RTT is defined as the ratio of the maximum to the 'second' maximum [8], or equivalently, it can be defined as the ratio of the 'second' maximum to the maximum, which can be expressed as

$$
\lambda=\frac{Y_{2}}{Y_{1}}, 0 \leq \lambda \leq 1 \text {. }
$$

Let $f_{\lambda}\left(r \mid H_{1}\right)$ and $f_{\lambda}\left(r \mid H_{0}\right)$ be the conditional PDFs under the hypotheses of $H_{1}$ associated with correct decisions and $H_{0}$ of erroneous decision of the MLD, respectively. The distributions of $f_{\lambda}\left(r \mid H_{1}\right)$ and $f_{\lambda}\left(r \mid H_{0}\right)$ using the same parameters as those in Fig. 3 are shown in Fig.4, when multipath Rayleigh fading channels were considered. According to Fig.4 we observe that $f_{\lambda}\left(r \mid H_{1}\right)$ is 


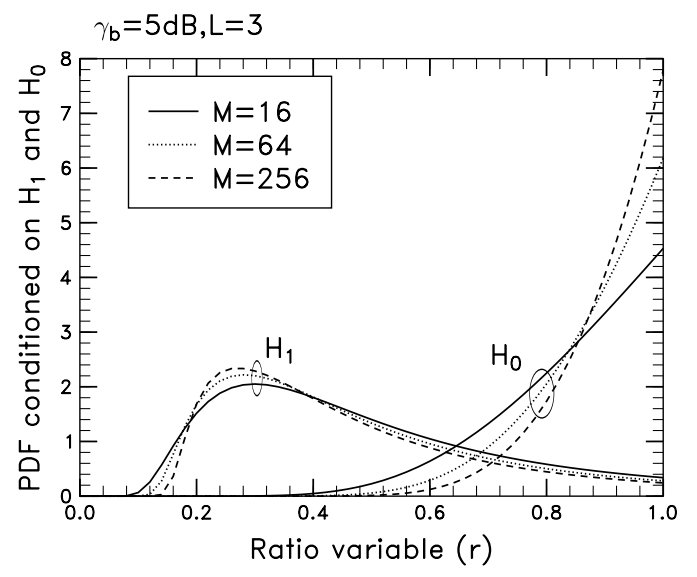

Fig. 4. The PDFs of $\lambda=\frac{Y_{2}}{Y_{1}}$ under the hypotheses of $H_{1}$ and $H_{0}$ using $M=16,64,256, L=3, \gamma_{b}=5 \mathrm{~dB}$ over multipath Rayleigh-fading channels.

mainly spread over the range having relatively low values of $\lambda$, while $f_{\lambda}\left(r \mid H_{0}\right)$ is mainly distributed over the range having relatively high values of $\lambda$. Therefore, the demodulated symbols having a relatively low ratio of $\lambda$ are more reliable, than those having relatively high values of $\lambda$. Consequently, a pre-set threshold $R_{T}$ can be invoked, in order to erase these low-reliability symbols associated with a ratio of $\lambda \geq R_{T}$.

\section{Joint MOR-TT}

In the context of the joint MOR-TT, the erasure insertion is based on the observation of both the maximum $Y_{1}$ of Eq.(4) and the ratio $\lambda$ of Eq.(6). Specifically, let $f_{Y_{1}, \lambda}\left(y, r \mid H_{1}\right)$ and $f_{Y_{1}, \lambda}\left(y, r \mid H_{0}\right)$ be the joint two-dimensional (2D) PDFs of the maximum $Y_{1}$ and the ratio $\lambda$ associated with the MOR-TT, conditioned on both the correct detection and erroneous detection of $M$-ary symbols. The properties of the MOR-TT can be studied with the aid of the 2D PDFs of $f_{Y_{1}, \lambda}\left(y, r \mid H_{1}\right)$ and $f_{Y_{1}, \lambda}\left(y, r \mid H_{0}\right)$, for example, which are shown in Fig.5 for the parameters of $M=64, L=3$ and $\gamma_{b}=5 \mathrm{~dB}$. From the 2D surfaces of Fig.5 we observe that for the given parameters the peak of the distribution $f_{Y_{1}, \lambda}\left(y, r \mid H_{1}\right)$ is located at a relatively high value of $Y_{1}$ or a relatively low value of $\lambda$, while the peak of the distribution $f_{Y_{1}, \lambda}\left(y, r \mid H_{0}\right)$ is located at a relatively low value of $Y_{1}$ and a relatively high value of $\lambda$. The above observations in turn imply that $f_{Y_{1}, \lambda}\left(y, r \mid H_{1}\right)$ is mainly spread over the range having relatively high values of $Y_{1}$ or relatively low values of $\lambda$, while $f_{Y_{1}, \lambda}\left(y, r \mid H_{0}\right)$ is mainly distributed over the range having relatively low values of $Y_{1}$ and relatively high values of $\lambda$. Therefore, if a demodulated symbol has a maximum output value of $Y_{1}$ and a ratio of $\lambda$, that had fallen in the main range of $f_{Y_{1}, \lambda}\left(y, r \mid H_{0}\right)$, the symbol concerned must be a low-reliability symbol and must be replaced by an erasure. By contrast, if a demodulated symbol has values of $Y_{1}$ and $\lambda$ that had fallen outside the main range of $f_{Y_{1}, \lambda}\left(y, r \mid H_{0}\right)$, then this symbol is likely to be correct and the corresponding RS code symbol can be forwarded to

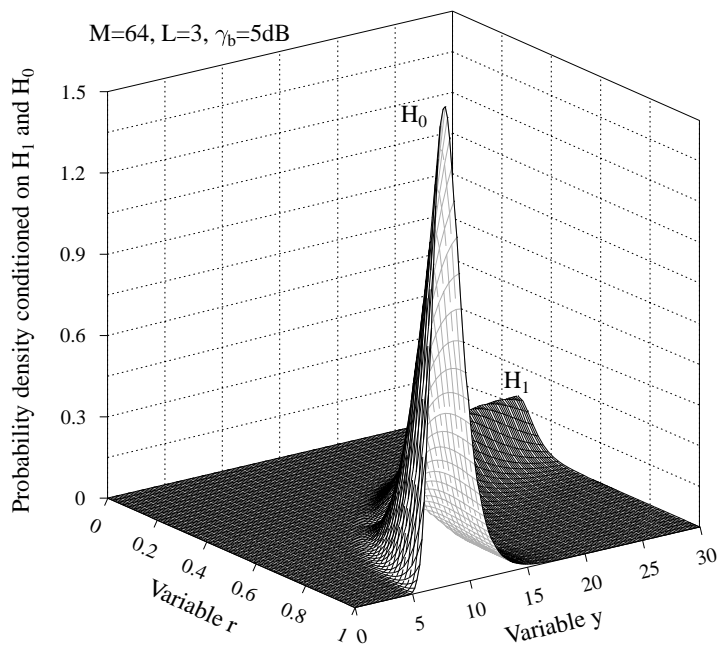

Fig. 5. The 2D joint PDFs of $f_{Y_{1}, \lambda}\left(y, r \mid H_{1}\right)$ and $f_{Y_{1}, \lambda}\left(y, r \mid H_{0}\right)$ using the parameters of $M=64, L=3, \gamma_{b}=5 \mathrm{~dB}$ over dispersive Rayleigh-fading channels.

the RS decoder. Consequently, in order to erase the lowreliability RS coded symbols, we assume that $Y_{T}$ and $R_{T}$ are two thresholds, which activate an erasure insertion, whenever $Y_{1} \leq Y_{T}$ and $\lambda \geq R_{T}$.

\section{D. $\mathbf{B T}$}

For $M$-ary orthogonal modulation systems using the Bayesian test, all the decision variables input to the MLD must be considered, in order to make an erasure decision. Specifically, let $s_{i}$ be the $i$ th code symbol of the alphabet, and $\mathbf{U}=\left(U_{1}^{i}, U_{2}^{i}, \ldots, U_{M}^{i}\right)$ be the vector of $M$-ary decision variables input to the MLD unit of Fig.2. Let $f\left(\mathbf{u} \mid s_{i}\right)$ be the conditional PDF of $\mathbf{U}$ given that $s_{i}$ was sent, and $\pi_{i}$ be the a priori probability of sending $s_{i}$. The $\mathrm{BT}$ decides that a received symbol should be erased if

$$
\frac{\max _{j} \pi_{j} f\left(\mathbf{u} \mid s_{j}\right)}{\sum_{i=1}^{M} \pi_{i} f\left(\mathbf{u} \mid s_{i}\right)} \leq(1-\theta),
$$

where $0<\theta<1$. Since in the BT-based erasure insertion scheme the PDFs of $f\left(\mathbf{u} \mid s_{i}\right)$ for $i=1,2, \ldots, M$ have to be estimated for making an erasure insertion decision, the complexity of the BT is significantly higher than that of the OTT, RTT or MOR-TT, which carry out an erasure decision based on the estimation of either $Y_{1}$ or $\lambda$, or both. Furthermore, the numerical results in [10] indicate that the BT-based erasure insertion scheme only slightly outperforms the RTT-based erasure insertion scheme, if the Signal to Interference plus noise ratio (SINR) is sufficiently high.

\section{E. Performance}

In this section the performance of RS coded $M$-ary CDMA using 'errors-and-erasures' decoding associated with the OTT, RTT and MOR-TT erasure insertion 


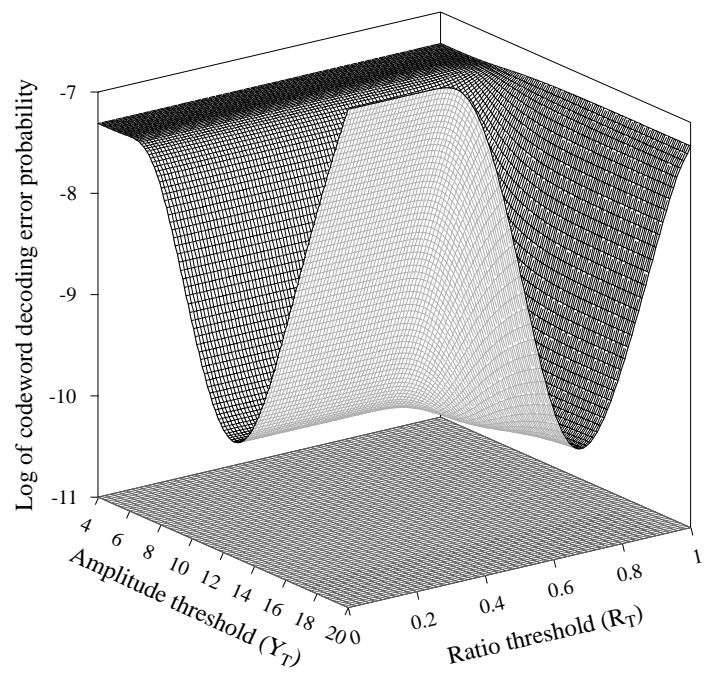

Fig. 6. Codeword decoding error probability versus the amplitude threshold, $Y_{T}$ and the ratio threshold, $R_{T}$ for the $\operatorname{RS}(32,20)$ FEC code using 'errors-and-erasures' decoding based on the MOR-TT erasure insertion scheme over dispersive Rayleigh fading channels using the parameters of $M=32, L=3, K=1$, $\gamma_{b}=12 \mathrm{~dB}$ and $\operatorname{RS}(32,20)$.

schemes is estimated and compared for a given set of parameters. Fig. 6 shows the codeword decoding error probability of the $M$-ary DS-CDMA system over dispersive Rayleigh fading channels using the joint MOR-TT. The $\mathrm{RS}(32,20)$ code constructed over the Galois Field $\mathrm{GF}(32)=\mathrm{GF}\left(2^{5}\right)$ corresponding to 5 -bit symbols was used and 'errors-and-erasures' decoding based on the MOR-TT insertion scheme was employed. From the results we observe that there exists an optimum threshold value of $R_{T}$ or $Y_{T}$, for which 'errors-and-erasures' decoding achieves the minimum codeword decoding error probability. This observation in turn implies that for given values of $M$, diversity order of $L$, number of users $K$, SNR per bit of $\gamma_{b}$ as well as for a given RS code, there exist optimum thresholds $Y_{T}$ and $R_{T}$, for which the 'errors-and-erasures' decoding using the joint MOR-TT erasure insertion scheme achieves the minimum codeword decoding error probability. This minimum codeword decoding error probability is lower, than that associated with using the RTT alone or the OTT alone. Note that in Fig.6 the point corresponding to $Y_{T}=0$ and $\lambda_{T}=1$ represents the codeword decoding error probability using 'error-correction-only' decoding. Therefore, we can observe that 'errors-and-erasures' decoding outperforms 'error-correction-only' decoding, if the appropriate thresholds are invoked. However, if the threshold $Y_{T}$ is too high and simultaneously the threshold $\lambda_{T}$ is too low, too many erasures will be activated, potentially erasing correct demodulated symbols. Consequently, the codeword decoding error probability using 'errors-and-erasures' decoding might be significantly higher, than that using 'error-correction-only' decoding.

Finally, in Fig.7 we compared the 'errors-and-erasures' RS decoding performance of our systems using the OTT, RTT and MOR-TT over dispersive Rayleigh fading chan-

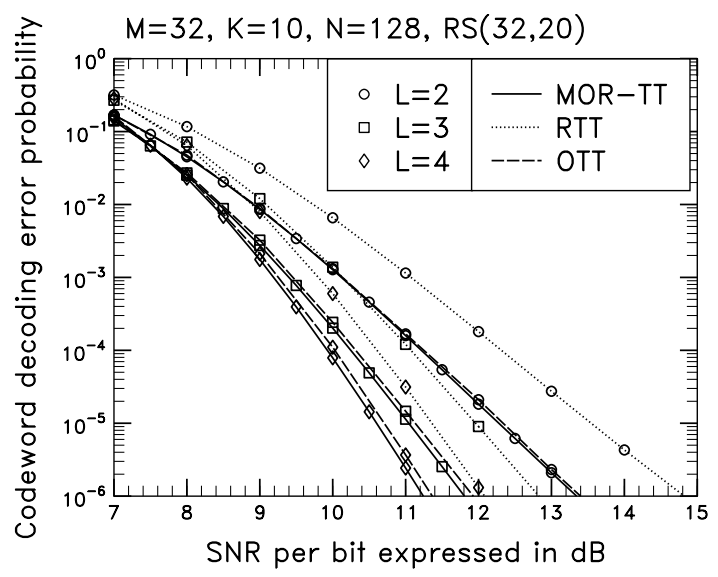

Fig. 7. Codeword decoding error probability versus SNR per bit for RS $(32,20)$ 'erasures-and-erasures' decoding using OTT, RTT and joint MOR-TT erasure insertion schemes.

nels. The parameters used was $M=32$, the number of users was $K=10$, the number of chips per bit was $N=128$ and the diversity order was $L=2,3,4$. From the results we observe that the proposed MOR-TT erasure scheme outperforms both the RTT and OTT erasure insertion schemes.

\section{REFERENCES}

[1] Telcomm. Industry Association (TIA), Washington, DC, Mobile station - Base station compatibility standard for dual-mode wideband spread spectrum cellular system, EIA/TIA Interim Standard IS-95, 1993.

[2] L. M. A. Jalloul and J. M. Holtzman, "Performance analysis of DS/CDMA with noncoherent $M$-ary orthogonal modulation in multipath fading channels," IEEE Journal on Selected Areas in Communications, vol. 12, pp. 862-870, June 1994

[3] S. W. Kim and W. Stark, "Performance limits of Reed-Solomon coded CDMA with orthogonal signaling in a Rayleigh-fading channel," IEEE Transactions on Communications, vol. 46, pp. 11251134, September 1998 .

[4] L.-L. Yang and L. Hanzo, "Errors-and-erasures decoding of ReedSolomon codes over frequency-selective Rayleigh fading channel using $M$-ary orthogonal signaling," in Proceedings of IEEE VTC'2000, (Tokyo, Japan), pp. 854-858, May 2000.

[5] L.-L. Yang, K. Yen, M. Dillinger, and L. Hanzo, "Performance of RS coded DS-CDMA using noncoherent $M$-ary orthogonal modulation over multipath fading channels," in Proceedings of PIMRC'2000, (London, UK), pp. 584-588, September 2000.

[6] L.-L. Yang and L. Hanzo, "Performance analysis of coded Mary orthogonal signaling using errors-and-erasures decoding over frequency-selective fading channels," To appear in IEEE Journal on Selected Areas in Communications.

[7] L.-L. Yang, K. Yen, and L. Hanzo, "A Reed-Solomon coded DSCDMA system using noncoherent M-ary orthogonal modulation over multipath fading channels," IEEE Journal on Selected Areas in Communications, vol. 18, November 2000

[8] A. J. Viterbi, "A robust ratio-threshold technique to mitigate tone and partial band jamming in coded MFSK systems," in Proceedings of IEEE Military Communications Conferences Rec., pp. 22.4.122.4.5, IEEE, October 1982.

[9] L.-L. Yang and L. Hanzo, "Performance analysis of RS coded DSCDMA using noncoherent $M$-ary orthogonal modulation over multipath fading channels," Submitted to IEEE Journal on Selected Areas in Communications, October 2000.

[10] C. W. Baum and M. B. Pursley, "Bayesian methods for erasure insertion in frequency-hop communication system with partialband interference," IEEE Transactions on Communications, vol. 40, pp. 1231-1238, July 1992 . 